\title{
BMJ Open Observational study of the differential impact of time-varying depressive symptoms on all-cause and cause- specific mortality by health status in community-dwelling adults: the REGARDS study
}

\author{
Nathalie Moise, ${ }^{1}$ Yulia Khodneva, ${ }^{2}$ Deanna Pereira Jannat-Khah, ${ }^{3}$ \\ Joshua Richman, ${ }^{2}$ Karina W Davidson, ${ }^{1}$ Ian M Kronish, ${ }^{1}$ Jonathan Shaffer, ${ }^{4}$ \\ Monika M Safford ${ }^{3}$
}

To cite: Moise N, Khodneva Y, Jannat-Khah DP, et al. Observational study of the differential impact of timevarying depressive symptoms on all-cause and cause-specific mortality by health status in community-dwelling adults: the REGARDS study. BMJ Open 2018;8:e017385. doi:10.1136/ bmjopen-2017-017385

- Prepublication history and additional material for this paper are available online. To view these files, please visit the journal online (http://dx.doi. org/10.1136/bmjopen-2017017385 )

Received 27 April 2017 Revised 20 November 2017 Accepted 21 November 2017

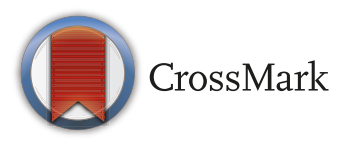

For numbered affiliations see end of article.

Correspondence to Dr Nathalie Moise; nm2562@cumc.columbia.edu

\section{ABSTRACT}

Objective To assess the association between time-varying depressive symptoms with all-cause and cause-specific mortality.

Design The REGARDS (Reasons for Geographic and Racial Differences in Stroke) is a national, population-based longitudinal study conducted from 2003 to 2007.

Setting General continental US communities.

Participants 29491 black and white US adults $\geq 45$ years randomly sampled within race-sex-geographical strata.

Exposure Elevated depressive symptoms (Centre for Epidemiologic Studies Depression (CES-D) 4 $\geq 4$ ) measured at baseline and on average 5 and 7 years later.

Main outcome measures Cox proportional hazard regression models assessed cancer, non-cardiovascular (cardiovascular disease (CVD)), CVD and all-cause mortality.

Results The average age was 64.9 years; $55 \%$ were women; $41 \%$ black; $11.0 \%$ had elevated depressive symptoms; $54 \%$ had poor, fair or good health. Timevarying depressive symptoms were significantly associated with non-CVD (adjusted HR (aHR) $=1.29,95 \% \mathrm{Cl}$ 1.16 to 1.44 ) and all-cause (aHR=1.24, $95 \% \mathrm{Cl} 1.14$ to 1.39), but not cancer ( $\mathrm{aHR}=1.15,95 \% \mathrm{Cl} 0.96$ to 1.38 ) or CVD (aHR=1.13, 95\% $\mathrm{Cl} 0.98$ to 1.32) death adjusting for demographics, chronic clinical diseases, behavioural risk factors and physiological factors. Depressive symptoms were related to all-cause $(\mathrm{aHR}=1.48,95 \% \mathrm{Cl}$ 1.27 to 1.78), CVD (aHR=1.37, 95\% $\mathrm{Cl} 0.99$ to 1.91), non-CVD (aHR=1.54, 95\% $\mathrm{Cl} 1.24$ to 1.92) and cancer (aHR=1.36, $95 \% \mathrm{Cl} 0.97$ to 1.91) death in those who reported excellent or very good health. The analyses of the association between one measure of baseline depressive symptoms and mortality analyses yielded similar results. Conclusions Time-varying depressive symptoms confer an increased risk for all-cause mortality, CVD, non-CVD death and cancer death, particularly in those with excellent or very good health. These findings may have implications for timely treatment, regardless of health status.

\section{Strengths and limitations of this study}

- Depression is a relapsing/remitting disease, and our study is one of the first to use multiple measurements of depression to demonstrate a time-varying relationship between depression and mortality, including cancer mortality, in a large, diverse cohort.

- To our knowledge, we are also the first to report a significant moderating effect of self-reported health on the relationship between depressive symptoms and cause-specific mortality, with depression predicting mortality particularly in those with excellent or very good reported health.

- Our analyses were limited by the use of the short form of the Centre for Epidemiologic Studies Depression scale.

- The REGARDS (Reasons for Geographic and Racial Differences in Stroke) cohort is regionally specific, limiting generalisability.

\section{INTRODUCTION}

It is well known that elevated depressive symptoms predict mortality, ${ }^{1}$ both in highrisk individuals with chronic illnesses such as cardiovascular disease (CVD) and in general populations. ${ }^{2-8}$ More recently, several studies have shown that depressive symptoms both preceding and following cancer diagnosis may confer an increased risk of cancer death as well. ${ }^{910}$

However, depressive symptoms relapse and remit, and prior studies on the relationship between depressive symptoms and mortality have been limited by one measurement of depressive symptoms. ${ }^{1}$ Recently, Lasserre et al found that current but not remitted 


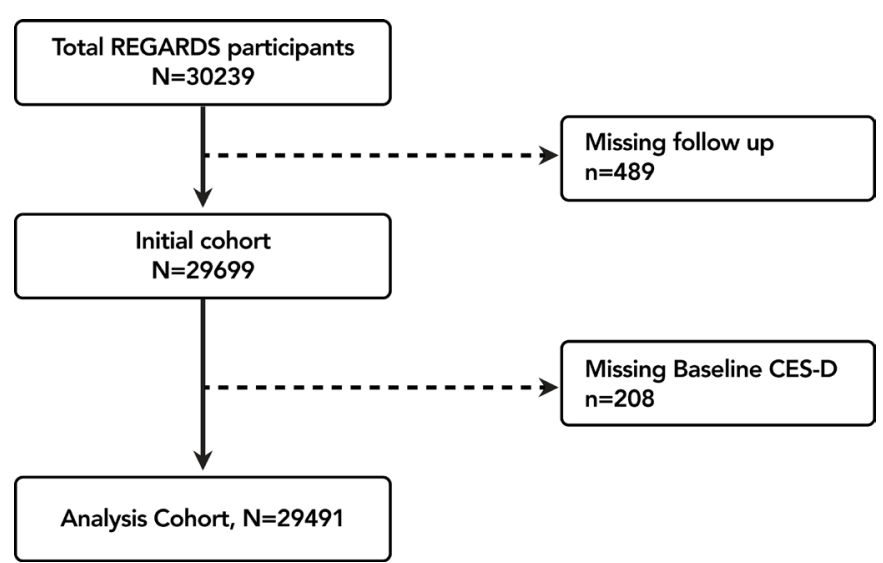

Figure 1 Cohort flow diagram: exclusion cascade of depressive symptoms to mortality endpoints analysis. CES-D, Centre for Epidemiologic Studies Depression; REGARDS, Reasons for Geographic and Racial Differences in Stroke .

depressive symptoms predict all-cause mortality, but again depression diagnoses and history were ascertained at one time point. ${ }^{11}$ In addition, prior literature has often been marked by inadequate adjustment for important covariates, such as behavioural risk factors. To our knowledge, few if any prior studies have examined the timevarying association between depressive symptoms and excess causes of death, including all-cause and cause-specific mortality. In addition, self-perceived health status may predict mortality ${ }^{12}$ and complicate the relationship between depressive symptoms and poor outcomes. ${ }^{13}$ It is unknown whether depressive symptoms confer an increased risk of excess mortality equally in those with self-reported excellent/very good (in whom depression may be less likely to be recognised) and good/fair/poor health.

The purpose of our study is to examine the association between time-varying depressive symptoms with cancer, CVD, non-CVD and all-cause mortality in the Reasons for Geographic and Racial Differences in Stroke (REGARDS) study, a broad, diverse population cohort with repeat measurements of depressive symptoms. We stratify by self-reported baseline health status (very good or excellent vs poor, fair or good) to further isolate the association between depressive symptoms and excess mortality.

\section{METHODS}

The REGARDS study is a national cohort study of stroke incidence and cognitive decline in black and white community-dwelling adults $\geq 45$ years living in the USA stratified to reflect specific race-sex-geographical strata. ${ }^{14}$ Inclusion and exclusion criteria have been previously described; of note, those with active cancer were excluded from the original study. ${ }^{14}$ Coronary heart disease (CHD) outcomes were ascertained from a REGARDS-MI ancillary study. Participants were recruited by mail using commercially available lists of US residents, followed by a computer-assisted telephone interview and subsequent home visit at which time individuals were consented and enrolled.
Between January 2003 and October 2007, 30239 black and white adults were enrolled. Of these, 489 (1.6\%) were lost to follow-up, and $208(0.7 \%)$ were missing baseline depressive symptom measurements (figure 1). The REGARDS study protocol was approved by institutional review boards at participating centres.

\section{Study procedures}

Baseline data were collected through computer-assisted telephone interviews, an in-home examination and self-administered questionnaires. Trained research staff conducted telephone interviews to collect demographic data, medical history and behavioural risk factors. Following the telephone interview, individuals had an in-home visit during which physical measurements, a resting ECG, medication inventory, phlebotomy and urine were collected. The median time between the initial phone interview and in-home examination was $28.0(\mathrm{IQR}=21.0)$ days.

\section{Primary outcomes}

The primary outcomes for these analyses were (1) cancer mortality (all body sites), (2) CVD death defined as death from CHD, stroke, heart failure, sudden cardiac death, vascular pathology and other CVD causes, (3) non-CVD death and (4) all-cause mortality. Living participants or their proxies were followed up every 6 months by telephone with retrieval of medical records for reported hospitalisations or physician visits. Deaths were detected by report of next-of-kin or through online services (eg, Social Security Death Index) or the National Death Index. ${ }^{14}$ Death certificates, medical records and autopsy reports were obtained to adjudicate cause of death and CVD outcomes.

\section{Depressive symptoms}

The primary predictor was baseline depressive symptoms. The 4-item Centre for Epidemiologic Studies Depression (CES-D) scale was used to assess the presence of depressive symptoms. This scale asks participants to rate the number of days over the last week in which they had: (1) felt depressed, (2) felt lonely, (3) had crying spells and (4) felt sad. Response options included $<1$ day, 1 to 2 days, 3 to 4 days and 5-7 days $(0,1,2$ and 3 points, respectively). Cronbach's $\alpha$ for the CES-D in the total sample was 0.80 . Elevated depressive symptoms were defined as a summed score of $\geq 4 .{ }^{15}$ The reliability and validity of the CES-D 4 is similar to the original 20-item instrument. ${ }^{16}$

\section{Covariates}

Demographic data included self-reported age, gender, race (black or white), education (less than high school, high school graduate, some college and college graduate and above), annual income (less than US\$20 000, US\$20 000-US\$34 999, US\$35 000-US\$74 999, US\$75000 and above), insurance status (yes/no) and stroke region (including the 'stroke belt' and 'stroke buckle'). Clinical risk factors included (1) diabetes defined as fasting blood glucose $\geq 126$ or random glucose $>200 \mathrm{mg} / \mathrm{dL}$ or oral 
Table 1A Overall baseline characteristics of REGARDS participants according to baseline depressive symptoms (CES-D)

\begin{tabular}{|c|c|c|c|c|}
\hline Characteristics & $\begin{array}{l}\text { Overall } \\
\text { (N=29491) }\end{array}$ & CES-D<4 $(n=26817)$ & $\begin{array}{l}\text { CES-D } \geq 4 \\
(n=3254)\end{array}$ & $\mathbf{P}$ \\
\hline \multicolumn{5}{|l|}{ Sociodemographics } \\
\hline Age, mean (SD) & $64.9(9.4)$ & $65.1(9.4)$ & $63.2(9.8)$ & $<0.001$ \\
\hline African-American, $\mathrm{n}(\%)$ & $12129(41.1)$ & $10427(39.7)$ & $1702(52.3)$ & $<0.001$ \\
\hline Less than high school education, $\mathrm{n}(\%)$ & 3696 (12.5) & $2916(11.1)$ & $780(24.0)$ & $<0.001$ \\
\hline Less than US $\$ 20000$ & $5322(18.0)$ & $4148(15.8)$ & $1174(36.1)$ & $<0.001$ \\
\hline No health insurance, $\mathrm{n}(\%)$ & $1926(6.5)$ & $1532(5.8)$ & $394(12.1)$ & $<0.001$ \\
\hline Region, n (\%) & & & & $<0.001$ \\
\hline Stroke belt ${ }^{*}$ & $10193(34.6)$ & $8973(34.2)$ & $1220(37.5)$ & \\
\hline Stroke buckle $†$ & $6188(21.0)$ & $5437(20.7)$ & $751(23.1)$ & \\
\hline Poor, fair, good & $15742(53.5)$ & $13219(50.5)$ & $2523(77.7)$ & \\
\hline Excellent, very good & $13690(46.5)$ & $12965(49.5)$ & $725(22.3)$ & \\
\hline CVD, n (\%)‡ & $6825(23.1)$ & $5838(22.3)$ & $987(30.3)$ & $<0.001$ \\
\hline Diabetes, $\mathrm{n}(\%) \S$ & $6252(22.0)$ & $5305(21.0)$ & $947(30.2)$ & $<0.001$ \\
\hline COPD, n (\%) & $2710(9.2)$ & $2307(8.8)$ & $403(12.4)$ & $<0.001$ \\
\hline Physical component score on SF-12 scale, mean (SD) & $46.4(10.6)$ & $47.1(10.2)$ & $40.7(12.2)$ & $<0.001$ \\
\hline \multicolumn{5}{|l|}{ Physiological risk factors } \\
\hline Body mass index, $\mathrm{kg} / \mathrm{m}^{2}$, mean (SD) & $29.3(6.2)$ & $29.2(6.1)$ & $30.6(7.1)$ & $<0.001$ \\
\hline Systolic blood pressure, mm Hg, mean (SD) & $127.6(16.7)$ & $127.5(16.5)$ & $128.7(18.1)$ & $<0.001$ \\
\hline Total cholesterol, mg/dL, mean (SD) & $192.1(40.1)$ & $191.7(39.8)$ & $194.6(43.0)$ & $<0.001$ \\
\hline Statin use, $n(\%)$ & $9295(31.6)$ & $8248(31.5)$ & $1047(32.3)$ & 0.38 \\
\hline Aspirin use, $\mathrm{n}(\%)$ & $12790(43.4)$ & $11376(43.4)$ & $1414(43.5)$ & 0.91 \\
\hline Antidepressant use, $\mathrm{n}(\%)$ & $4086(13.9)$ & $3164(12.1)$ & $922(28.4)$ & $<0.001$ \\
\hline \multicolumn{5}{|l|}{ Behavioural risk factors } \\
\hline Self-reported smoking, pack-years, mean (SD) & $13.5(23.1)$ & $13.3(22.8)$ & $15.5(24.9)$ & $<0.001$ \\
\hline Current smoking, $\mathrm{n}(\%)$ & $4263(14.5)$ & $3463(13.3)$ & $800(24.7)$ & $<0.001$ \\
\hline Alcohol use, $\mathrm{n}(\%)$ & & & & $<0.001$ \\
\hline Heavy & $1172(4.1)$ & $1043(4.0)$ & $129(4.1)$ & \\
\hline Moderate & $9626(33.3)$ & $8786(34.1)$ & $840(26.6)$ & \\
\hline None & $18116(62.7)$ & $15925(61.8)$ & $2191(69.3)$ & \\
\hline Physical inactivity, n (\%) & $10004(34.4)$ & $8500(32.9)$ & $1504(46.9)$ & $<0.001$ \\
\hline Medication non-adherence, $\mathrm{n}(\%)$ & $7959(29.7)$ & $6820(28.7)$ & $1139(37.8)$ & $<0.001$ \\
\hline Impaired cognitive status (cognitive score $\leq 4$ ) & $1888(7.9)$ & $1542(7.3)$ & $346(12.6)$ & $<0.001$ \\
\hline Elevated perceived stress (PSS $\geq 5$ ) & $8591(29.1)$ & $6283(23.9)$ & 2308 (70.9) & $<0.001$ \\
\hline
\end{tabular}

$P$ values from $\chi^{2}$ and Student's t-tests.

$\mathrm{n}=$ total number assuming no missing data.

*Stroke belt defined as the states of Alabama, Arkansas, Louisiana, Mississippi and Tennessee and the non-coastal regions within the states of North Carolina,

South Carolina and Georgia.

†Stroke buckle defined as the coastal regions within the states of North Carolina, South Carolina and Georgia.

$\ddagger C V D$ defined as baseline coronary heart disease, stroke, peripheral artery disease or aortic aneurysm.

$\S$ Diabetes defined as fasting blood glucose $\geq 126$ or random glucose $>200 \mathrm{~mL} / \mathrm{dL}$ or oral hypoglycaemic or insulin use.

CES-D, Centre for Epidemiology Studies Depression scale; COPD, chronic obstructive pulmonary disease; CVD, cardiovascular disease; PSS, Perceived Stress

Scale; REGARDS, Reasons for Geographic and Racial Differences in Stroke; SF-12, Short-Form Health Survey. 
hypoglycaemic or insulin use, (2) systolic and diastolic blood pressures based on the average of two standardised blood pressure measurements (in $\mathrm{mm} \mathrm{Hg}$ ), (3) body mass index (BMI) based on measured height and weight, (4) albumin to creatinine ratio (logarithmically-transformed), (5) high-density lipoprotein (HDL)-cholesterol, (6) total cholesterol, (7) history of CVD: CHD (self-reported history of myocardial infarction or coronary revascularisation procedure or evidence of myocardial infarction on the study ECG), self-reported stroke, peripheral vascular disease or aneurysm, (8) cognitive impairment on the 6-item screener of global cognitive function ${ }^{17} 18$ and (9) chronic lung disease defined as use of beta- 2 adrenergic agonists, leukotriene inhibitors, inhaled corticosteroids, combination inhalers or other pulmonary medications such as ipratropium, cromolyn, aminophylline and theophylline. We also assessed self-reported (yes/no) aspirin, antidepressant (serotonin and norepinephrine reuptake inhibitors, selective serotonin reuptake inhibitors and tricyclic antidepressants), statin and antihypertensive use. Behavioural risk factors included (1) self-reported pack-years of cigarette smoking, (2) physical activity ('How many times per week do you engage in intense physical activity, enough to work up a sweat?' with response options of 'none', '1-3 times per week' and ' 4 or more times per week'), (3) alcohol use ('How many alcoholic beverages do you drink?': none, moderate (one drink per day for women or two drinks per day for men) and heavy (greater than one drink per day for women and two drinks per day for men) $)^{14}$ and (4) medication non-adherence assessed with the 4-item Morisky Medication Adherence Scale $(\geq 1) \cdot{ }^{19}$ Potential physiological risk factors included high-sensitivity $\mathrm{C}$ reactive protein, self-reported health status based on the physical component of the 12-item Short-Form Health Survey $(\mathrm{SF} 12)^{20}$ and perceived stress, measured by the 4 -item version of the Perceived Stress Scale (score of $\geq 5$ vs $<5$ ). ${ }^{21}$ Other than depressive symptoms, no other covariate was assessed more than once.

\section{Statistical analyses}

Baseline characteristics of participants with and without elevated depressive symptoms at baseline were compared using $\chi^{2}$ tests (for categorical variables), Student's t-tests (for continuous variables) and Wilcoxon rank-sum tests (for non-normally distributed continuous measures).

Cox proportional hazard regression models were constructed to separately analyse the association between depressive symptoms (CES-D $\geq 4$ ) and cancer death (from all body sites, a subset of non-CVD death), CVD death, non-CVD death and all-cause death. The end date of follow-up for this analysis was 31 December 2012. Depressive symptoms were measured on the CES-D scale: (1) at baseline (initial telephone call), (2) on average 5 years after baseline measurement and (3) on average 2 years after the second measurement. In the analyses, we considered depressive symptoms (CES-D $\geq 4$ vs $<4$ ) as a timevarying exposure, with updates of exposure at 5 year and
7 year follow-up. Therefore, each participant contributed up to three measures of CES-D ( $\geq 4$ vs $<4$ ) with a broken-up follow-up time. Follow-up time for each participant was calculated from the date of the in-home visit to the date of the earliest of: death, last telephone follow-up, end of follow-up or next CES-D measure. We additionally graphically plotted unadjusted survival functions for participants with elevated versus non-elevated depressive symptoms using the Simon-Makuch method, ${ }^{22}$ a modification of the Kaplan-Meier method. In this context, depression status is treated as a binary time-dependent covariate, and study cohorts are continually updated to contribute to either the CES-D $\geq 4$ or CES-D $<4$ groups.

Unadjusted HRs and 95\% CIs of mortality endpoints were estimated for those with versus without elevated depressive symptoms. Adjusted modelling proceeded in stages (adjusting for baseline covariates), starting with demographic (model 1) and traditional CVD risk factors (model 2) assessed in prior trials. We then added behavioural (model 3) and other potential explanatory (model 4) factors. We also ran an additional model (model 5), which considered intervening first non-fatal stroke and/or myocardial infarction as a time-dependent covariate in CVD death outcomes. All analyses were conducted overall as well as stratified. We also conducted a formal test for interaction between depressive symptoms and self-reported health (defined as excellent or very good versus good, fair or poor health) in model 4 . As such, all analyses were conducted overall as well as stratified by baseline self-reported health. To evaluate the possibility of non-proportional hazards, we graphically inspected the log-log survival plots for depressive symptoms. We tested the Schoenfeld residuals for each model for a non-zero slope, and all $\mathrm{P}$ values were greater than 0.05 , indicating compatibility with the proportional hazards assumption.

\section{Sensitivity analyses}

Sensitivity analyses constructed in parallel to the main analyses examined association of baseline CES-D measure with mortality endpoints in the sequentially-adjusted Cox proportional hazard regression models. The end date of follow-up for this analysis was 31 December 2012. Follow-up time for each participant was calculated from the date of the in-home visit to the date of the earliest of: death, last telephone follow-up or end of follow-up.

Missing data in covariates were imputed using chained equations and derived by bootstrapping across the five imputed datasets. Multiple imputation was used for all analyses. Of the 29491 participants, 2768 (9\%) were missing income data, $59(0.2 \%)$ health status, 9 $(<0.1 \%)$ education, $26(0.1 \%)$ health insurance, 1087 $(4 \%)$ diabetes, $16(0.1 \%)$ aspirin use, $70(0.2 \%)$ statin use, $70(0.2 \%)$ antidepressant use, $333(1 \%)$ antihypertension medicines use, 439 (2\%) physical activity, 2705 (9\%) medication adherence, $213(0.7 \%)$ BMI, $1254(4 \%)$ cholesterol, 1401 (5\%) HDL, $912(3.1 \%)$ pack-years, 84 $(0.3 \%)$ systolic blood pressure, $1394(5 \%)$ renal function, 
Table 1B Baseline characteristics of REGARDS participants according to baseline depressive symptoms (CES-D) and selfreported health

\begin{tabular}{|c|c|c|c|c|c|c|}
\hline \multirow[b]{2}{*}{ Characteristics } & \multicolumn{3}{|c|}{$\begin{array}{l}\text { Self-reported general health as } \\
\text { 'excellent or very good' }\end{array}$} & \multicolumn{3}{|c|}{$\begin{array}{l}\text { Self-reported general health as 'poor } \\
\text { fair or good' }\end{array}$} \\
\hline & $\begin{array}{l}\text { CES-D<4 } \\
(\mathrm{N}=12965)\end{array}$ & $\begin{array}{l}\text { CES-D } \geq 4 \\
(\mathrm{~N}=725)\end{array}$ & $\mathbf{P}$ & $\begin{array}{l}\text { CES-D<4 } \\
(\mathrm{N}=13219)\end{array}$ & $\begin{array}{l}\text { CES-D } \geq 4 \\
(\mathrm{~N}=2523)\end{array}$ & $\mathbf{P}$ \\
\hline \multicolumn{7}{|l|}{ Sociodemographics } \\
\hline Age, mean (SD) & $64.8(9.4)$ & $64.5(10.2)$ & 0.47 & $65.5(9.3)$ & $62.8(9.6)$ & $<0.001$ \\
\hline Female, n (\%) & $6600(50.9)$ & $501(69.1)$ & $<0.001$ & $7357(55.7)$ & $1751(69.4)$ & $<0.001$ \\
\hline African-American, n (\%) & $3726(28.7)$ & $295(40.7)$ & $<0.001$ & $6677(50.5)$ & $1404(55.6)$ & $<0.001$ \\
\hline Less than high school education, $\mathrm{n}(\%)$ & $845(6.5)$ & $119(16.4)$ & $<0.001$ & 2059 (15.6) & $658(26.1)$ & $<0.001$ \\
\hline \multicolumn{7}{|l|}{ Annual household income, $\mathrm{n}(\%)$} \\
\hline Less than US $\$ 20000$ & $1304(10.1)$ & $190(26.2)$ & $<0.001$ & $2832(21.4)$ & $983(39.0)$ & $<0.001$ \\
\hline No health insurance, $\mathrm{n}(\%)$ & $644(5.0)$ & $70(9.7)$ & $<0.001$ & $884(6.7)$ & $324(12.9)$ & $<0.001$ \\
\hline Region, n (\%) & & & 0.37 & & & $<0.001$ \\
\hline Stroke belt ${ }^{\star}$ & $4282(33.0)$ & $256(35.3)$ & & $4668(35.3)$ & $963(38.2)$ & \\
\hline Stroke buckle† & 2619 (20.2) & $148(20.4)$ & & $2807(21.2)$ & $601(23.8)$ & \\
\hline Non-stroke belt or buckle & $6064(46.8)$ & $321(44.3)$ & & $5744(43.5)$ & $959(38.0)$ & \\
\hline \multicolumn{7}{|l|}{ General health and medical conditions } \\
\hline \multicolumn{7}{|l|}{ Self-reported general health, $\mathrm{n}(\%)$} \\
\hline Poor, fair, good & - & - & & - & - & \\
\hline Excellent, very good & - & - & & - & - & \\
\hline CVD, n (\%)‡ & $1948(15.0)$ & $144(19.9)$ & 0.004 & $3874(29.3)$ & $840(33.3)$ & $<0.001$ \\
\hline Diabetes, n (\%)§ & $1443(11.6)$ & 93 (13.3) & 0.16 & $3840(30.2)$ & $853(35.1)$ & $<0.001$ \\
\hline COPD, n (\%) & $796(6.2)$ & $55(7.6)$ & 0.11 & $1507(11.4)$ & $347(13.8)$ & 0.007 \\
\hline $\begin{array}{l}\text { Physical component score on SF-12 } \\
\text { scale, mean (SD) }\end{array}$ & $52.0(6.5)$ & $51.3(9.1)$ & 0.008 & $42.0(10.7)$ & $37.7(11.3)$ & $<0.001$ \\
\hline \multicolumn{7}{|l|}{ Physiological risk factors } \\
\hline Body mass index, $\mathrm{kg} / \mathrm{m}^{2}$, mean (SD) & $27.8(5.1)$ & $28.4(5.7)$ & 0.006 & $30.5(6.6)$ & $31.2(7.3)$ & $<0.001$ \\
\hline $\begin{array}{l}\text { Systolic blood pressure, } \mathrm{mm} \mathrm{Hg} \text {, mean } \\
\text { (SD) }\end{array}$ & $125.3(15.7)$ & $126.0(17.2)$ & 0.27 & $129.6(16.9)$ & $129.5(18.3)$ & 0.91 \\
\hline Total cholesterol, mg/dL, mean (SD) & $193.8(38.2)$ & $195.5(38.6)$ & 0.26 & $189.7(41.2)$ & $194.4(44.2)$ & $<0.001$ \\
\hline $\begin{array}{l}\text { High-density lipoprotein, mg/dL, mean } \\
\text { (SD) }\end{array}$ & $53.1(16.4)$ & $55.8(16.6)$ & $<0.001$ & $50.4(15.8)$ & $51.5(16.1)$ & 0.002 \\
\hline $\begin{array}{l}\text { QT interval, corrected for heart rate, } \\
\text { ms, mean (SD) }\end{array}$ & $405.6(22.6)$ & $407.2(23.5)$ & 0.06 & $408.7(24.3)$ & $410.8(24.2)$ & $<0.001$ \\
\hline $\begin{array}{l}\text { High-sensitivity C reactive protein, } \\
\text { mg/L, median, IQR }\end{array}$ & $1.7(0.8-3.8)$ & $1.9(0.9-4.9)$ & 0.004 & $2.7(1.2-6.1)$ & $3.4(1.3-7.7)$ & $<0.001$ \\
\hline $\begin{array}{l}\text { Albumin to creatinine ratio, } \mathrm{mg} / \mathrm{g} \text {, } \\
\text { median, IQR }\end{array}$ & $6.6(4.3-12.3)$ & $6.9(4.7-14.0)$ & 0.005 & $8.4(5.0-20.7)$ & $8.7(5.1-22.2)$ & 0.18 \\
\hline \multicolumn{7}{|l|}{ Medications } \\
\hline Antihypertensive medication use, $\mathrm{n}(\%)$ & $4916(38.3)$ & $297(41.7)$ & 0.06 & $8344(63.9)$ & $1606(64.5)$ & 0.57 \\
\hline Statin use, $\mathrm{n}(\%)$ & $3407(26.4)$ & $176(24.4)$ & 0.24 & $4822(36.5)$ & $870(34.6)$ & 0.06 \\
\hline Aspirin use, $\mathrm{n}(\%)$ & $5254(40.5)$ & $273(37.7)$ & 0.13 & $6100(46.2)$ & $1140(45.2)$ & 0.36 \\
\hline Antidepressant use, $\mathrm{n}(\%)$ & $1224(9.5)$ & $144(19.9)$ & $<0.001$ & $1933(14.6)$ & $774(30.8)$ & $<0.001$ \\
\hline \multicolumn{7}{|l|}{ Behavioural risk factors } \\
\hline $\begin{array}{l}\text { Self-reported smoking, pack-years, } \\
\text { mean (SD) }\end{array}$ & $11.2(20.5)$ & $12.1(21.6)$ & 0.24 & $15.3(24.7)$ & $16.5(25.6)$ & 0.03 \\
\hline Current smoking, n (\%) & $1344(10.4)$ & $114(15.8)$ & $<0.001$ & $2110(16.0)$ & $684(27.2)$ & $<0.001$ \\
\hline
\end{tabular}




\begin{tabular}{|c|c|c|c|c|c|c|}
\hline \multirow[b]{2}{*}{ Characteristics } & \multicolumn{3}{|c|}{$\begin{array}{l}\text { Self-reported general health as } \\
\text { 'excellent or very good' }\end{array}$} & \multicolumn{3}{|c|}{$\begin{array}{l}\text { Self-reported general health as 'poor, } \\
\text { fair or good' }\end{array}$} \\
\hline & $\begin{array}{l}\text { CES-D<4 } \\
(\mathrm{N}=12965)\end{array}$ & $\begin{array}{l}\text { CES-D } \geq 4 \\
(\mathrm{~N}=725)\end{array}$ & $\mathbf{P}$ & $\begin{array}{l}\text { CES-D }<4 \\
(\mathrm{~N}=13219)\end{array}$ & $\begin{array}{l}\text { CES-D } \geq 4 \\
(\mathrm{~N}=2523)\end{array}$ & $\mathbf{P}$ \\
\hline Alcohol use, n (\%) & & & 0.01 & & & $<0.001$ \\
\hline Heavy & $634(5.0)$ & $38(5.4)$ & & 409 (3.2) & $91(3.7)$ & \\
\hline Moderate & $5034(39.4)$ & $238(33.8)$ & & $3746(29.0)$ & $600(24.5)$ & \\
\hline None & $7103(55.6)$ & $429(60.9)$ & & $8779(67.9)$ & $1758(71.8)$ & \\
\hline Physical inactivity, $\mathrm{n}(\%)$ & 3107 (24.3) & $259(36.0)$ & $<0.001$ & $5372(41.3)$ & $1242(50.0)$ & $<0.001$ \\
\hline Medication non-adherence, n (\%) & $2997(26.2)$ & $211(33.1)$ & $<0.001$ & $3809(31.0)$ & $926(39.1)$ & $<0.001$ \\
\hline $\begin{array}{l}\text { Impaired cognitive status } \\
\text { (cognitive score } \leq 4 \text { ) }\end{array}$ & $587(5.6)$ & $61(10.1)$ & $<0.001$ & $947(8.9)$ & $285(13.3)$ & $<0.001$ \\
\hline Elevated perceived stress (PSS $\geq 5$ ) & $2219(17.1)$ & $404(55.7)$ & $<0.001$ & $4048(30.6)$ & $1900(75.3)$ & $<0.001$ \\
\hline
\end{tabular}

$P$ values from $\chi^{2}$ and Student' s t-tests.

$\mathrm{n}=$ total number assuming no missing data.

*Stroke belt defined as the states of Alabama, Arkansas, Louisiana, Mississippi and Tennessee and the non-coastal regions within the states of North Carolina, South Carolina and Georgia.

†Stroke buckle defined as the coastal regions within the states of North Carolina, South Carolina and Georgia.

¥CVD defined as baseline coronary heart disease, stroke, peripheral artery disease or aortic aneurysm.

§Diabetes defined as fasting blood glucose $\geq 126$ or random glucose $>200 \mathrm{~mL} / \mathrm{dL}$ or oral hypoglycaemic or insulin use.

CES-D, Centre for Epidemiology Studies Depression scale; COPD, chronic obstructive pulmonary disease; CVD, cardiovascular disease;

PSS, Perceived Stress Scale; REGARDS, Reasons for Geographic and Racial Differences in Stroke; SF-12, Short-Form Health Survey.

$381(1 \%)$ corrected QT, $5681(19.3 \%)$ cognitive status, $4(<0.1 \%)$ stress, $1425(4 \%)$ SF-12 and $1881(6 \%)$ CRP. Analyses were conducted using SAS software V.9.4 and STATA V.12.

\section{RESULTS}

\section{Participant characteristics}

Overall, $1.6 \%$ were lost to follow-up, and $0.7 \%$ were missing baseline depressive symptoms, leaving 29491 eligible participants (figure 1) of whom $3254(11.0 \%)$ had elevated depressive symptoms at baseline (CES-D $\geq 4)$. The average age was 64.9 (9.4) years; $55.1 \%$ were women, $41.1 \%$ were black, $22.0 \%$ had diabetes, $9.2 \%$ chronic lung disease and $23.1 \%$ CVD. Nearly $33 \%$ of individuals were physically inactive, $29.2 \%$ non-adherent to their medication regimen and $14.5 \%$ current smokers. A total of $53.5 \%$ of participants self-reported their general health to be poor, fair or good compared with $46.5 \%$ who reported their health to be excellent or very good, of whom $16.0 \%$ and $5.3 \%$ had elevated depressive symptoms, respectively (online supplementary etable 1). Regardless of health status, participants with elevated (vs non-elevated) depressive symptoms were more likely to be women, African-American, low income, have more chronic diseases, low physical health and more behavioural risk factors (table 1A,B).

\section{Mortality}

A total of $4581(15.5 \%)$ participants died during the follow-up period ending in 2012. Of these, 1551 (33.9\%) were attributed to CVD, and $3030(66.1 \%)$ to non-CVD disease death. Of non-CVD deaths, $1226(44.3 \%)$ were due to cancer death (online supplementary etable 2). Overall, there were only three cases of mortality due to suicide.

For the time-varying analyses, depressive symptoms were measured at baseline and on average 4.8 (SD 1.5) years following the baseline measurement, the third measurement occurring on average 2.1 (SD 0.4) years after the second measurement (online supplementary efigure 1). The mean follow-up time of the second and third measurements of CES-D measures did not differ by self-reported health (online supplementary efigure 2). Of the participants with elevated depressive symptoms at baseline, $39.9 \%$ and $36.8 \%$ had elevated depressive symptoms at the second and third measures, respectively (online supplementary etable 3 ). Timevarying depressive symptoms significantly predicted non-CVD disease death (aHR 1.29, 95\% CI 1.16 to 1.44) and all-cause mortality (adjusted HR (aHR) 1.24, $95 \%$ CI 1.14 to 1.36 ), while approaching significance for cancer death (aHR 1.15, 95\% CI 0.96 to 1.38) and CVD death (aHR 1.13, 05\% CI 0.98 to 1.32 ), even after adjusting for demographic, clinical, behavioural and physiological factors and time-varying non-fatal CVD events (table 2, online supplementary efigure 3). The results appeared to be particularly robust among those with excellent or very good self-reported general health: all-cause (aHR $=1.48,95 \%$ CI 1.27 to 1.78$)$, CVD $(\mathrm{aHR}=1.37,95 \%$ CI 0.99 to 1.91$)$, non-CVD $(\mathrm{aHR}=1.54$, 95\% CI 1.24 to 1.92$)$ and cancer $(\mathrm{aHR}=1.36,95 \%$ CI 0.97 to 1.91 ) death. In model 4 , the $\mathrm{P}$ values for the 
Table 2 Association of elevated depressive symptoms with mortality outcomes. Each participant contributes up to three timevariant CES-D measures

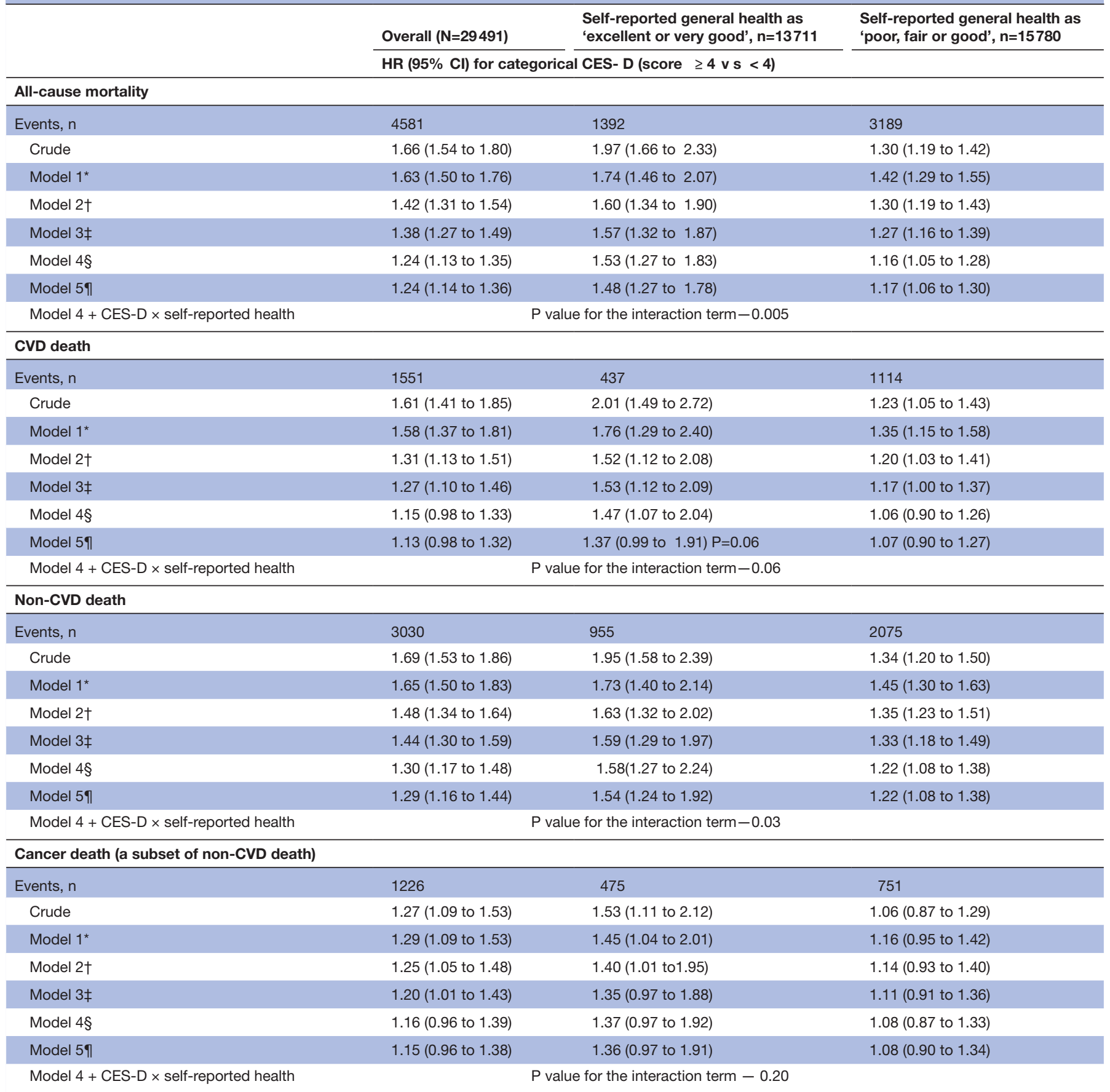

End of follow-up is 31 December 2012. Bold $\mathrm{P}$ value $<0.05$; missing data in covariates imputed using chained equations.

*Model 1 adjusts for sociodemographics (age, gender, region, income, health insurance and education).

†Model 2 adds to model 1 medical conditions, physiological factors and medication use (systolic blood pressure, total cholesterol, high-density lipoprotein-

cholesterol, use of aspirin, statins, antihypertensives, antidepressants, body mass index, logarithmically transformed albumin to creatinine ratio, diabetes, CVD, medication use as a proxy for chronic obstructive pulmonary disease and cognitive impairment).

¥Model 3 adds to model 2 behavioural risk factors (pack-years of cigarette smoking, self-reported alcohol use, physical inactivity and medication non-adherence). $\S$ Model 4 adds to model 3 other factors (physical health component score of SF-12, log-transformed high sensitivity $\mathrm{C}$ reactive protein and perceived stress).

IModel 5 adds non-fatal CVD event-first non-fatal myocardial infarction or stroke since baseline.

CES-D, Centre for Epidemiology Studies Depression; CVD, cardiovascular disease; SF-12, Short-Form Health Survey.

depressive symptoms $\times$ health status interaction term were 0.005 (all-cause mortality), 0.06 (CVD death), 0.03 (non-CVD death) and 0.20 (cancer death). Results were similar without multiple imputations within two decimal places (table 2).

\section{Sensitivity analyses}

The mean follow-up time was $6.5(\mathrm{SD}=2.3)$ years, with a median (IQR) of 6.9 (5.4-8.3) years. Baseline depressive symptoms were significantly associated with all-cause mortality (aHR $1.18,95 \%$ CI 1.07 to 1.29 ) and non-CVD 
death (aHR 1.21, 95\% CI 1.08 to 1.36 ) and approached significance for CVD death (aHR 1.10, 95\% CI 0.94 to 1.29 ) and cancer death (aHR 1.12, 95\% CI 0.93 to 1.36), even in the exploratory models (model 3). The results appeared to be particularly robust among those with excellent or very good health: cancer death (aHR 1.49, 95\% CI 1.03 to 2.13), CVD death (aHR 1.63, 95\% CI 1.16 to 2.30 ), non-CVD death (aHR $1.48,95 \%$ CI 1.15 to 1.89 ) and all-cause mortality (aHR $1.53,95 \%$ CI 1.25 to 1.88 ). In model 4 , the $\mathrm{P}$ values for depressive symptoms $\times$ health status interaction term were 0.003 (all-cause mortality), 0.01 (CVD death), 0.06 (non-CVD death) and 0.07 (cancer death). Results were similar without multiple imputations within two decimal places (table 3).

\section{DISCUSSION}

To our knowledge, this is the largest study to date to examine the relationship between depressive symptoms and all-cause and cause-specific mortality in non-institutionalised middle to older aged adults using multiple measurements of depressive symptoms and examining the role of health status. In this diverse cohort, we found that time-varying depressive symptoms significantly increased the risk of non-CVD and all-cause mortality in fully adjusted models. In fully adjusted models, depressive symptoms increased the risk of cause-specific and all-cause mortality by $36 \%$ to $54 \%$ in those with a very good/excellent state of health.

Given that depression is a relapsing/remitting disease, ${ }^{23}$ this study markedly adds to the literature by demonstrating a time-varying relationship between elevated depressive symptoms and mortality, including cancer death. Major study strengths include the use of three measurements of depressive symptoms and stringent physician adjudication of outcomes. We were, however, unable to adjust for other time-varying covariates, which should be addressed in future research. For example, prior research suggests that changes in physical health (eg, number of debilitating conditions) over time may mediate the relationship between depressive symptoms and mortality. ${ }^{24}$

We are also the first to report a significant moderating effect of self-reported health on the relationship between depressive symptoms and mortality. Many have long asked whether depression leads to mortality or whether individuals are depressed because they are dying. Our findings in those who report excellent states of health are striking and support the former argument. It may also be that the effect of chronic illness burden on mortality in those with poor health overwhelms the effects of depressive symptoms. Those with excellent health may also fail to recognise/present for depression. In fact, depressed excellent health individuals in our cohort were less likely to be on an antidepressant. Nonetheless, this finding should be further explored in future studies.

The overall results also have a coherence consistent with prior studies that suggest that depressive symptoms don't solely predict suicide and CVD mortality, but also predict other causes such as cancer death. ${ }^{25}$ While prior literature suggests that depressive symptoms confer mortality in those with active cancer, ${ }^{26}$ our study excluded active cancer diagnoses confirming a possible relationship between depressive symptoms and incident cancer mortality. Prior studies have also been limited by inadequate covariate control, and our results for cancer persisted after adjusting for numerous traditional and behavioural risk factors, such as smoking, and approached significance even in models that included physiological factors.

Overall, baseline and time-varying analyses were similar. However, while our baseline analyses suggest that depressive symptoms significantly contribute to cancer death in those with excellent/very good health, time-varying analyses allowed for more accurate analyses in line with expectations, suggesting a weaker interaction by health status for proximal cancer mortality in this cohort that excluded those with active malignancy.

This study also supports comprehensive evidence-based depression care management in primary care practices, which have been shown to lower mortality risk. ${ }^{27}$ Nonetheless, depression treatment remains suboptimal in the general population, ${ }^{28}$ despite decades of efforts. We too demonstrate that over time, nearly $40 \%$ of patients with elevated depressive symptoms at baseline were still depressed on average 5 and 7 years later. Given the potentially shorter follow-up times in both time-varying analyses (by virtue of follow-up times being broken up by repeat depression measures) and baseline analyses (with 6.5 years of follow-up on average), these findings lend greater urgency to the importance of timely and effective treatment of depressive symptoms to prevent adverse consequences of depressive symptoms on physical health and mortality.

Limitations of our study include the regional specificity, limiting generalisability and use of the short form of the CES-D, which measures only emotional and not somatic symptoms of depression. Schultz et al demonstrated variance between studies using scales and interviews, ${ }^{29}$ and others have posited even stronger findings in studies with clinical diagnoses (vs continuous measures) ${ }^{30}$ However, CES-D scales are one of the most widely used scales in clinical practice and in baseline depression to outcome studies and have good sensitivity and specificity. ${ }^{91516}$ We may also have been underpowered to examine CVD and cancer mortality, though the directionality of the estimates remained consistent. The exclusion of active cancer participants as part of the overall REGARDS study criteria, the rationale of which has previously been described ${ }^{14}$ may also have contributed to lack of power. Those with a history of malignancy or CVD were not specifically excluded, which is in line with prior depression to mortality studies. ${ }^{19}$ Nonetheless, our previously published study, which excluded those with a history of CVD, similarly found a strong relationship between time-varying depressive symptoms and CVD death. ${ }^{31}$ 
Table 3 Association of baseline only elevated depressive symptoms (CES-D $\geq 4$ ) with mortality outcomes

\begin{tabular}{|c|c|c|c|}
\hline & Overall $N=29491$ & $\begin{array}{l}\text { Self-reported } \\
\text { general health as } \\
\text { 'excellent or very } \\
\text { good', } n=13711\end{array}$ & $\begin{array}{l}\text { Self-reported } \\
\text { general health as } \\
\text { 'poor, fair or good', } \\
n=15780\end{array}$ \\
\hline & HR (95\% Cl) & HR (95\% Cl) & HR $(95 \% \mathrm{Cl})$ \\
\hline All-cause mortality & 4581 & 1392 & 3189 \\
\hline Crude & 1.54 (1.42 to 1.68$)$ & 1.91 (1.59 to 2.31$)$ & 1.18 (1.07 to 1.30$)$ \\
\hline Model $1^{*}$ & 1.57 (1.44 to 1.72$)$ & 1.76 (1.45 to 2.12$)$ & 1.34 (1.21 to 1.47$)$ \\
\hline Model 2† & $1.32(1.25$ to 1.49$)$ & 1.61 (1.33 to 1.96$)$ & $1.22(1.11$ to 1.35$)$ \\
\hline Model 3ł & $1.32(1.27$ to 1.44$)$ & 1.56 (1.29 to 1.90$)$ & 1.20 (1.09 to 1.32$)$ \\
\hline Model $4 \S$ & 1.18 (1.07 to 1.29$)$ & 1.53 (1.25 to 1.88$)$ & 1.09 (0.98 to 1.20$)$ \\
\hline Model $4+$ baseline CES-D $\times$ self-reported health & \multicolumn{3}{|c|}{$P$ value for the interaction term -0.002} \\
\hline CVD death & 1551 & 437 & 1114 \\
\hline Crude & 1.55 (1.34 to 1.78$)$ & 2.16 (1.58 to 2.96$)$ & $1.13(0.97$ to 1.33$)$ \\
\hline Model $1^{*}$ & 1.57 (1.35 to 1.81$)$ & 1.96 (1.42 to 2.71$)$ & 1.29 (1.10 to 1.52$)$ \\
\hline Model $2 \dagger$ & $1.28(1.10$ to 1.48$)$ & 1.71 (1.23 to 2.38$)$ & 1.14 (0.97 to 1.34$)$ \\
\hline Model $3 \ddagger$ & $1.24(1.07$ to 1.44$)$ & 1.70 (1.22 to 2.36$)$ & $1.11(0.94$ to 1.31$)$ \\
\hline Model $4 \S$ & $1.10(0.94$ to 1.29$)$ & $1.63(1.16$ to 2.30$)$ & $1.00(0.84$ to 1.20$)$ \\
\hline Model $4+$ baseline CES-D $\times$ self-reported health & \multicolumn{3}{|c|}{$P$ value for the interaction term -0.01} \\
\hline Non-CVD death & 3030 & 955 & 2075 \\
\hline Crude & $1.54(1.39$ to 1.71$)$ & $1.80(1.42$ to 2.26$)$ & 1.21 (1.08 to 1.35$)$ \\
\hline Model $1^{*}$ & $1.57(1.42$ to 1.75$)$ & 1.66 (1.31 to 2.10$)$ & 1.36 (1.21 to 1.53$)$ \\
\hline Model $2 \dagger$ & 1.41 (1.26 to 1.56$)$ & 1.56 (1.29 to 1.98$)$ & 1.27 (1.13 to 1.43$)$ \\
\hline Model $3 \ddagger$ & $1.36(1.22$ to 1.51$)$ & 1.49 (1.17 to 1.90$)$ & 1.25 (1.11 to 1.41$)$ \\
\hline Model 4§ & 1.21 (1.08 to 1.36$)$ & $1.48(1.15$ to 1.89$)$ & 1.14 (1.00 to 1.29$)$ \\
\hline Model $4+$ baseline CES-D $\times$ self-reported health & \multicolumn{3}{|c|}{$P$ value for the interaction term -0.06} \\
\hline Cancer death (a subset of non-CVD death) & 1226 & 475 & 751 \\
\hline Crude & $1.21(1.02$ to 1.44$)$ & 1.63 (1.16 to 2.30$)$ & 0.97 (0.79 to 1.19$)$ \\
\hline Model $1^{*}$ & 1.27 (1.06 to 1.52$)$ & 1.58 (1.12 to 2.23$)$ & 1.09 (0.89 to 1.35$)$ \\
\hline Model $2 \dagger$ & $1.22(1.02$ to 1.47$)$ & 1.53 (1.08 to 2.17$)$ & 1.07 (0.87 to 1.33$)$ \\
\hline Model 3ł & 1.17 (0.98 to 1.41$)$ & 1.45 (1.02 to 2.05$)$ & 1.05 (0.85 to 1.30$)$ \\
\hline Model $4 \S$ & $1.12(0.93$ to 1.36$)$ & 1.49 (1.03 to 2.13$)$ & $1.01(0.81$ to 1.27$)$ \\
\hline Model $4+$ baseline CES-D $\times$ self-reported health & \multicolumn{3}{|c|}{$P$ value for the interaction term -.07} \\
\hline
\end{tabular}

*Model 1 adjusts for sociodemographics (age, gender, region, income, health insurance and education).

†Model 2 adds to model 1 medical conditions, physiological factors and medication use (systolic blood pressure, total cholesterol, high-density lipoprotein-cholesterol, use of aspirin, statins, antihypertensives, antidepressants, body mass index, logarithmically transformed albumin to creatinine ratio, diabetes, CVD, medication use as a proxy for chronic obstructive pulmonary disease and cognitive impairment).

¥Model 3 adds to model 2 behavioural risk factors (pack-years of cigarette smoking, self-reported alcohol use, physical inactivity and medication non-adherence).

§Model 4 adds to model three other factors (physical health component score of SF-12, log-transformed high sensitivity $\mathrm{C}$ reactive protein and perceived stress).

Each participant contributes one measure of CES-D at baseline.

CES-D, Centre for Epidemiology Studies Depression; CVD, cardiovascular disease; SF-12, Short-Form Health Survey . $\mathrm{HR}$ and $95 \% \mathrm{Cl}$ were estimated by Cox proportional hazard regression models. Bold $\mathrm{P}$ value $<0.05$; missing data in covariates imputed using chained equations.

We were also unable to adjust for other psychiatric comorbidities, such as anxiety (though we included stress) or account for subclinical CVD and/or cancer. In addition, the follow-up time (6.5 years) was relatively short compared with other studies, and we saw even shorter follow-up times between CES-D measures in time-varying 
analyses, suggesting a short-term effect on mortality. Our results support prior literature suggesting that shorter follow-up time is associated with greater excess mortality. ${ }^{930}$ However, we did not formally compare shortterm to long-term follow-up nor persistent to fluctuating depressive symptoms nor examine depression as a timevarying coefficient.

Given our results of a relationship between time-varying depressive symptoms and mortality, further research is warranted to test the long-term efficacy of and adherence to depression treatment and to explore preventive approaches to decreasing premature mortality risk. ${ }^{32}$ To our knowledge, the finding of a relationship between depressive symptoms and mortality in those with excellent or very good self-reported health is a new finding and should be further studied.

\section{Author affiliations}

${ }^{1}$ Center for Behavioral Cardiovascular Health, Department of Medicine, Columbia University Medical Center, New York, New York, USA

${ }^{2}$ University of Alabama School of Medicine, Birmingham, Alabama, USA

${ }^{3}$ Department of Medicine, Weill Cornell Medical College, New York, New York, USA

${ }^{4}$ Department of Psychology, University of Colorado Denver, Denver, Colorado, USA

Acknowledgements The authors thank the other investigators, the staff and the participants of the REGARDS study for their valuable contributions.

Contributors YK, JR and DPJ-K had full access to all the data in the study and take responsibility for the integrity of the data and the accuracy of the data analysis. Study concept and design: NM, YK and JS. Acquisition of data: YK and JS. Analysis and interpretation of data: YK, NM, DPJ-K, JR, IMK, JS and MMS. Drafting of the manuscript: NM and YK. Critical revision of the manuscript for important intellectual content: NM, YK, DPJ-K, JR, IMK, KWD, JS and MMS. Statistical analysis: YK and DPJ-K. Obtained funding and study supervision: MMS.

Funding This research project is supported by a cooperative agreement U01 NS041588 from the National Institute of Neurological Disorders and Stroke, National Institutes of Health and Department of Health and Human Service. The REGARDS study was supported by NIH grant 2U01NS041588. The REGARDS-MI study was supported by NIH grants R01 HL080477 and K24 HL111154. A full list of participating REGARDS investigators and institutions can be found at http:// www.regardsstudy.org. This work was also supported by funds from NHLBI (3 R01 HL114924-03S1, HL080477 and K24 HL111154).

Competing interests None declared.

Ethics approval Institutional review boards at participating centers.

Provenance and peer review Not commissioned; externally peer reviewed.

Data sharing statement Patient level data or full dataset or technical appendix or statistical code is available if deemed important by reviewers with open access by MMS at Weill Cornell, NM at Columbia University Medical Center and YK at University of Alabama at Birmingham.

Open Access This is an Open Access article distributed in accordance with the Creative Commons Attribution Non Commercial (CC BY-NC 4.0) license, which permits others to distribute, remix, adapt, build upon this work non-commercially, and license their derivative works on different terms, provided the original work is properly cited and the use is non-commercial. See: http://creativecommons.org/ licenses/by-nc/4.0/

(c) Article author(s) (or their employer(s) unless otherwise stated in the text of the article) 2018. All rights reserved. No commercial use is permitted unless otherwise expressly granted.

\section{REFERENCES}

1. Wulsin LR, Vaillant GE, Wells VE. A systematic review of the mortality of depression. Psychosom Med 1999;61:6-17.
2. Sullivan MD, O'Connor P, Feeney P, et al. Depression predicts allcause mortality: epidemiological evaluation from the ACCORD HRQL substudy. Diabetes Care 2012;35:1708-15.

3. Huffman JC. Review: depression after myocardial infarction is associated with increased risk of all-cause mortality and cardiovascular events. Evid Based Ment Health 2013;16:110.

4. Barth J, Schumacher M, Herrmann-Lingen C. Depression as a risk factor for mortality in patients with coronary heart disease: a metaanalysis. Psychosom Med 2004;66:802-13.

5. Cuijpers P, Vogelzangs N, Twisk J, et al. Comprehensive meta-analysis of excess mortality in depression in the general community versus patients with specific illnesses. Am J Psychiatry 2014;171:453-62.

6. Cuijpers P, Smit F. Excess mortality in depression: a meta-analysis of community studies. J Affect Disord 2002;72:227-36.

7. Bruce ML, Leaf PJ, Rozal GP, et al. Psychiatric status and 9-year mortality data in the New Haven Epidemiologic Catchment Area Study. Am J Psychiatry 1994;151:716-21.

8. Surtees PG, Wainwright NW, Luben RN, et al. Depression and ischemic heart disease mortality: evidence from the EPIC-Norfolk United Kingdom prospective cohort study. Am J Psychiatry 2008;165:515-23.

9. Pinquart M, Duberstein PR. Depression and cancer mortality: a meta-analysis. Psychol Med 2010;40:1797-810.

10. Mols F, Husson O, Roukema JA, et al. Depressive symptoms are a risk factor for all-cause mortality: results from a prospective population-based study among 3,080 cancer survivors from the PROFILES registry. J Cancer Surviv 2013;7:484-92.

11. Lasserre AM, Marti-Soler H, Strippoli MP, et al. Clinical and course characteristics of depression and all-cause mortality: a prospective population-based study. J Affect Disord 2016;189:17-24.

12. McGee DL, Liao Y, Cao G, et al. Self-reported health status and mortality in a multiethnic US cohort. Am J Epidemiol 1999;149:41-6.

13. Ambresin G, Chondros P, Dowrick C, et al. Self-rated health and long-term prognosis of depression. Ann Fam Med 2014;12:57-65.

14. Howard VJ, Cushman M, Pulley L, et al. The reasons for geographic and racial differences in stroke study: objectives and design. Neuroepidemiology 2005;25:135-43.

15. Melchior LA, Huba GJ, Brown VB, et al. A short depression index for women. Educ Psychol Meas 1993;53:1117-25.

16. Radloff LS. The ces-d scale: a self-report depression scale for research in the general population. Appl Psychol Meas 1977:1:385-401.

17. Folstein MF, Folstein SE, McHugh PR. "Mini-mental state". A practical method for grading the cognitive state of patients for the clinician. J Psychiatr Res 1975;12:189-98.

18. Callahan CM, Unverzagt FW, Hui SL, et al. Six-item screener to identify cognitive impairment among potential subjects for clinical research. Med Care 2002;40:771-81.

19. Morisky DE, Green LW, Levine DM. Concurrent and predictive validity of a self-reported measure of medication adherence. Med Care 1986;24:67-74

20. Ware J, Kosinski M, Keller SD. A 12-Item short-form health survey: construction of scales and preliminary tests of reliability and validity. Med Care 1996;34:220-33.

21. Cohen S, Kamarck T, Mermelstein R. A global measure of perceived stress. J Health Soc Behav 1983;24:385-96.

22. Simon R, Makuch RW. A non-parametric graphical representation of the relationship between survival and the occurrence of an event: application to responder versus non-responder bias. Stat Med 1984:3:35-44.

23. Lin EH, Katon WJ, VonKorff M, et al. Relapse of depression in primary care. Rate and clinical predictors. Arch Fam Med 1998;7:443-9.

24. Houle JN. Depressive symptoms and all-cause mortality in a nationally representative longitudinal study with time-varying covariates. Psychosom Med 2013;75:297-304.

25. Mykletun A, Bjerkeset O, Dewey M, et al. Anxiety, depression, and cause-specific mortality: the HUNT study. Psychosom Med 2007;69:323-31.

26. Satin JR, Linden W, Phillips MJ. Depression as a predictor of disease progression and mortality in cancer patients: a meta-analysis. Cancer 2009;115:5349-61.

27. Gallo JJ, Morales KH, Bogner HR, et al. Long term effect of depression care management on mortality in older adults: followup of cluster randomized clinical trial in primary care. BMJ 2013;346:f2570.

28. González HM, Vega WA, Williams DR, et al. Depression care in the United States: too little for too few. Arch Gen Psychiatry 2010;67:37-46. 
29. Schulz R, Drayer RA, Rollman BL. Depression as a risk factor for non-suicide mortality in the elderly. Biol Psychiatry 2002;52:205-25.

30. Pan A, Sun Q, Okereke Ol, et al. Depression and risk of stroke morbidity and mortality: a meta-analysis and systematic review. JAMA 2011;306:1241-9.

31. Moise N, Khodneva Y, Richman J, et al. Elucidating the association between depressive symptoms, coronary heart disease, and stroke in black and white adults: the reasons for geographic and racial differences in stroke (REGARDS) study. J Am Heart Assoc 2016;5:e003767.

32. Kuyken W, Warren FC, Taylor RS, et al. Efficacy of mindfulnessbased cognitive therapy in prevention of depressive relapse: an individual patient data meta-analysis from randomized trials. JAMA Psychiatry 2016;73:565-74. 